TRANSACTIONS OF THE

AMERICAN MATHEMATICAL SOCIETY

Volume 361, Number 11, November 2009, Pages 6151-6163

S 0002-9947(09)04794-1

Article electronically published on June 24, 2009

\title{
THE LEFSCHETZ PROPERTY FOR BARYCENTRIC SUBDIVISIONS OF SHELLABLE COMPLEXES
}

\author{
MARTINA KUBITZKE AND ERAN NEVO
}

\begin{abstract}
We show that an 'almost strong Lefschetz' property holds for the barycentric subdivision of a shellable complex. From this we conclude that for the barycentric subdivision of a Cohen-Macaulay complex, the $h$-vector is unimodal, peaks in its middle degree (one of them if the dimension of the complex is even), and that its $g$-vector is an $M$-sequence. In particular, the (combinatorial) $g$-conjecture is verified for barycentric subdivisions of homology spheres. In addition, using the above algebraic result, we derive new inequalities on a refinement of the Eulerian statistics on permutations, where permutations are grouped by the number of descents and the image of 1 .
\end{abstract}

\section{INTRODUCTION}

The starting point for this paper is Brenti and Welker's study of $f$-vectors of barycentric subdivisions of simplicial complexes [3. They showed that for a Cohen-Macaulay complex, the $h$-vector of its barycentric subdivision is unimodal ([3, Corollary 3.5]). This raises the following natural questions about this $h$-vector: Where is its peak? Is the vector of its successive differences up to the middle degree (' $g$-vector') an $M$-sequence?

We answer these questions by finding an 'almost strong Lefschetz' element in case the original complex is shellable. Let us make this precise (for unexplained terminology see Section 2): let $\Delta$ be a $(d-1)$-dimensional Cohen-Macaulay simplicial complex over a field $k$, on a vertex set $[n]:=\{1, \ldots, n\}$ and $\Theta=\left\{\theta_{1}, \ldots, \theta_{d}\right\}$ a linear system of parameters for its face ring $k[\Delta]$. We call a degree one element in the polynomial ring $\omega \in A=k\left[x_{1}, \ldots, x_{n}\right]$ an $s$-Lefschetz element for the A-module $k[\Delta] / \Theta$ if multiplication

$$
\omega^{s-2 i}:(k[\Delta] / \Theta)_{i} \longrightarrow(k[\Delta] / \Theta)_{s-i}
$$

is an injection for $0 \leq i \leq\left\lfloor\frac{s-1}{2}\right\rfloor$. A $(\operatorname{dim} \Delta)$-Lefschetz element is called an almost strong Lefschetz element for $k[\Delta] / \Theta$. (Recall that $k[\Delta] / \Theta$ is called strong Lefschetz if it has a $(\operatorname{dim} \Delta+1)$-Lefschetz element.) Let $G_{s}(\Delta)$ be the set consisting of all pairs $(\Theta, \omega)$ such that $\Theta$ is a linear system of parameters for $k[\Delta]$ and $\omega$ is an $s$-Lefschetz element for $k[\Delta] / \Theta$. It can be shown that $G_{s}(\Delta)$ is a Zariski open set

Received by the editors January 25, 2008 and, in revised form, March 18, 2008

2000 Mathematics Subject Classification. Primary 13F55.

Key words and phrases. Barycentric subdivision, Stanley-Reisner ring, Lefschetz, shellable.

The first author was supported by DAAD. 
(e.g., imitate the proof in [15, Proposition 3.6]). If $G_{s}(\Delta) \neq \emptyset$ we say that $\Delta$ is $s$-Lefschetz over $k$, and that $\Delta$ is almost strong Lefschetz over $k$ if $G_{\operatorname{dim} \Delta}(\Delta) \neq \emptyset$.

Theorem 1.1. Let $\Delta$ be a shellable $(d-1)$-dimensional simplicial complex and let $k$ be an infinite field. Then the barycentric subdivision of $\Delta$ is almost strong Lefschetz over $k$.

This theorem has some immediate $f$-vector consequences; in particular it verifies the $g$-conjecture for barycentric subdivisions of homology spheres, and beyond. One of the main problems in algebraic combinatorics is the $g$-conjecture, first raised as a question by McMullen for simplicial spheres [8]. Here we state the part of the conjecture which is still open.

Conjecture 1.2 ( $g$-conjecture). Let $L$ be a simplicial sphere. Then its g-vector is an $M$-sequence.

It is conjectured to hold in greater generality, for all homology spheres and even for all doubly Cohen-Macaulay complexes, as was suggested by Björner and Swartz [15. We verify these conjectures in a special case, as was already conjectured in [2]:

Corollary 1.3. Let $\Delta$ be a Cohen-Macaulay simplicial complex (over some field). Then the g-vector of the barycentric subdivision of $\Delta$ is an $M$-sequence. In particular, the g-conjecture holds for barycentric subdivisions of simplicial spheres, of homology spheres, and of doubly Cohen-Macaulay complexes.

In Section 2 we provide some preliminaries and prove our main result, Theorem 1.1. In Section 3 we derive some $f$-vector corollaries from Theorem 1.1, as well as extending this theorem to shellable polytopal complexes. In Section 4 we prove new inequalities for the refined Eulerian statistics on permutations, introduced by Brenti and Welker. The proofs are based on Theorem 1.1. As a corollary, the location of the peak of the $h$-vector of the barycentric subdivision of a Cohen-Macaulay complex is determined.

\section{Proof of Theorem 1.1}

Let $\Delta$ be a finite (abstract, non-empty) simplicial complex on a vertex set $\Delta_{0}=$ $[n]=\{1,2, \ldots, n\}$, i.e., $\Delta \subseteq 2^{[n]}$ and if $S \subseteq T \in \Delta$, then $S \in \Delta$ (and $\emptyset \in \Delta$ ),

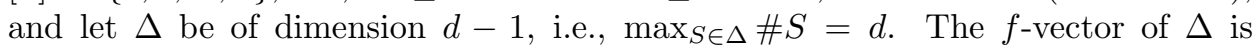
$f^{\Delta}=\left(f_{-1}, f_{0}, \ldots, f_{d-1}\right)$, where $f_{i-1}=\#\{S \in \Delta \mid \# S=i\}$. Its $h$-vector, which carries the same combinatorial information as the $f$-vector, is $h^{\Delta}=\left(h_{0}, \ldots, h_{d}\right)$, where $\sum_{0 \leq i \leq d} h_{i} x^{d-i}=\sum_{0 \leq i \leq d} f_{i-1}(x-1)^{d-i}$.

Let $k$ be an infinite field and $A=k\left[x_{1}, \ldots, x_{n}\right]=A_{0} \oplus A_{1} \oplus \ldots$ the polynomial ring graded by degree. The face ring (Stanley-Reisner ring) of $\Delta$ over $k$ is $k[\Delta]=A / I_{\Delta}$, where $I_{\Delta}$ is the ideal $I_{\Delta}=\left(\prod_{1 \leq i \leq n} x_{i}^{a_{i}} \mid\left\{i \mid a_{i}>0\right\} \notin \Delta\right) . k[\Delta]$ inherits the grading from $A$. If $k$ is infinite, a homogeneous system of parameters for $k[\Delta]$ can be chosen from the 'linear' part $k[\Delta]_{1}$, called an l.s.o.p. for short. If $k[\Delta]$ is CohenMacaulay (CM for short) and $\Theta=\left\{\theta_{1}, \ldots, \theta_{d}\right\}$ is any l.s.o.p., then $k[\Delta]$ is a free $k[\Theta]$-module, and $h_{i}^{\Delta}=\operatorname{dim}_{k}(k[\Delta] / \Theta)_{i}$. (Throughout we use $\Theta$ to denote both the l.s.o.p. and the ideal generated by it, depending on the context.) We say that $\Delta$ is $\mathrm{CM}$ (over $k$ ) if $k[\Delta]$ is a CM ring.

The barycentric subdivision of a simplicial complex $\Delta$ is the simplicial complex $\operatorname{sd}(\Delta)$ on the vertex set $\Delta \backslash\{\emptyset\}$ whose simplices are all the chains $F_{0} \subsetneq F_{1} \subsetneq \ldots \subsetneq F_{r}$ 
of elements $F_{i} \in \Delta \backslash\{\emptyset\}$ for $0 \leq i \leq r$. The geometric realizations of $\Delta$ and $\operatorname{sd}(\Delta)$ are homeomorphic. Recall that Cohen-Macaulayness is a topological property. Hence, if $\Delta$ is $\mathrm{CM}$, then $\operatorname{sd}(\Delta)$ is $\mathrm{CM}$ as well. In this case Baclawski and Garsia (1, Proposition 3.4]) showed that $\Theta=\left\{\theta_{1}, \ldots, \theta_{\operatorname{dim} \Delta+1}\right\}$, where $\theta_{i}:=\sum_{F \in \Delta, \# F=i} x_{\{F\}}$ for $1 \leq i \leq \operatorname{dim} \Delta+1$ is an 1.s.o.p. for $k[\operatorname{sd}(\Delta)]$. For further terminology and background we refer to Stanley's book [14. Let us start with some auxiliary results.

We denote by cone $(\Delta)$ the cone over $\Delta$; i.e., $\operatorname{cone}(\Delta)$ is the join of some vertex $\{v\}$ with $\Delta$, where $v \notin \Delta$, cone $(\Delta):=\{F \mid F \in \Delta\} \cup\{F \cup\{v\} \mid F \in \Delta\}$. The following lemma deals with the effect of coning on the $s$-Lefschetz property.

Lemma 2.1. Let $\Delta$ be a $(d-1)$-dimensional simplicial complex. If $\Delta$ is s-Lefschetz over $k$, then the same is true for $\operatorname{cone}(\Delta)$.

Proof. Let $\Theta$ be an 1.s.o.p. for $k[\Delta]$ and let $v$ be the apex of $\operatorname{cone}(\Delta)$. Then $\widetilde{\Theta}:=\Theta \cup\left\{x_{v}\right\}$ is an l.s.o.p. for $k[\operatorname{cone}(\Delta)]$ (to see this, use the isomorphism $k[\operatorname{cone}(\Delta)] \cong k[\Delta] \otimes_{k} k\left[x_{v}\right]$ of modules over $k\left[x_{u} \mid u \in \Delta_{0} \cup\{v\}\right] \cong k\left[x_{u} \mid u \in\right.$ $\left.\left.\Delta_{0}\right] \otimes_{k} k\left[x_{v}\right]\right)$. Furthermore, $k[\Delta] / \Theta \cong k[\operatorname{cone}(\Delta)] / \widetilde{\Theta}$ as $A$-modules, where $A=$ $k\left[x_{i} \mid i \in\{v\} \cup \Delta_{0}\right]$ and $x_{v} \cdot k[\Delta]=0$. Hence, for any pair $(\Theta, \omega) \in G_{s}(\Delta)$ we have $(\widetilde{\Theta}, \omega) \in G_{s}(\operatorname{cone}(\Delta))$, and the assertion follows.

Note that if $\Delta$ is almost strong Lefschetz over $k$, then $\operatorname{cone}(\Delta)$ is $(\operatorname{dim} \Delta)$ Lefschetz over $k$.

The following theorem is the main part of Stanley's proof of the necessity part of the $g$-theorem for simplicial polytopes [13].

Theorem 2.2 ([13]). Let $P$ be a simplicial d-polytope and let $\Delta$ be the boundary complex of $P$. Then $\Delta$ is d-Lefschetz over $\mathbb{R}$.

If $\Delta$ is a simplicial complex and $\left\{F_{1}, \ldots, F_{m}\right\} \subseteq \Delta$ is a collection of faces of $\Delta$ we denote by $\left\langle F_{1}, \ldots, F_{m}\right\rangle$ the simplicial complex whose faces are the subsets of the $F_{i}$ 's, $1 \leq i \leq m$.

For an arbitrary infinite field $k$ (of arbitrary characteristic!) the conclusion in Theorem 2.2 holds for the following polytopes. This fact will suffice to derive our main result, Theorem 1.1

Proposition 2.3. Let $P$ be a d-simplex and let $\Delta$ be its barycentric subdivision. Let $k$ be an infinite field. Then $\Delta$ is almost strong Lefschetz over $k$.

Proof. Note that the boundary complex $\partial \Delta$ is obtained from $\partial P$ by a sequence of stellar subdivisions: order the faces of $\partial P$ by decreasing dimension and perform a stellar subdivision at each of them according to this order to obtain $\partial \Delta$. In particular, $\partial \Delta$ is strongly edge decomposable. This can be seen by using the inverse stellar moves starting with the last simplicial complex in the sequence of complexes and moving backwards. The notion of strongly edge decomposable complexes was introduced in [10].

It was shown by Murai [9, Corollary 3.5] that strongly edge decomposable complexes have the strong Lefschetz property (see also [11, Corollary 4.6.6]). As $\Delta=\operatorname{cone}(\partial \Delta)$, we conclude that $\Delta$ is $d$-Lefschetz over $k$ by Lemma 2.1 .

We would like to point out that the proof of Proposition 2.3 is self-contained and does not require Theorem 2.2. Shellability of simplicial complexes is a useful tool in combinatorics; here we give two equivalent definitions for shellability which we will use later. 
Definition 2.4. A pure simplicial complex $\Delta$ is called shellable if $\Delta$ is a simplex or if one of the following equivalent conditions is satisfied. There exists a linear ordering $F_{1}, \ldots, F_{m}$ of the facets of $\Delta$ such that

(a) $\left\langle F_{i}\right\rangle \cap\left\langle F_{1}, \ldots, F_{i-1}\right\rangle$ is generated by a non-empty set of maximal proper faces of $\left\langle F_{i}\right\rangle$, for all $2 \leq i \leq m$.

(b) The set $\left\{F \mid F \in\left\langle F_{1}, \ldots, F_{i}\right\rangle, F \notin\left\langle F_{1}, \ldots, F_{i-1}\right\rangle\right\}$ has a unique minimal element for all $2 \leq i \leq m$. This element is called the restriction face of $F_{i}$. We denote it by $\operatorname{res}\left(F_{i}\right)$.

A linear order of the facets satisfying the equivalent conditions (a) and (b) is called a shelling of $\Delta$.

We are now in a position to prove Theorem 1.1. In the sequel, we will loosely use the term 'generic elements' to mean that these elements are chosen from a non-empty Zariski open set, to be understood from the context.

Proof of Theorem 1.1. The proof is by double induction, on the number of facets $f_{\operatorname{dim} \Delta}^{\Delta}$ of $\Delta$ and on the dimension of $\Delta$. If $\Delta$ is a complex of an arbitrary dimension that has only one top-dimensional face, then $\Delta$ is a simplex, and we are done by Proposition 2.3. Let $\operatorname{dim} \Delta=0$, i.e., $\Delta$ as well as $\operatorname{sd}(\Delta)$ consists of vertices only. Since $h_{0}^{\operatorname{sd}(\Delta)}=h_{1-1-0}^{\operatorname{sd}(\Delta)}$ there is nothing to show. This provides the base of induction.

For the induction step let $\operatorname{dim} \Delta \geq 1$. Let $n=f_{0}^{\operatorname{sd}(\Delta)}$ and let $A=k\left[x_{1}, \ldots, x_{n}\right]$ be the polynomial ring in $n$ variables. Let $F_{1}, \ldots, F_{m}$ be a shelling of $\Delta$ with $m \geq 2$ and let $\widetilde{\Delta}:=\left\langle F_{1}, \ldots, F_{m-1}\right\rangle$. Then $\sigma:=\widetilde{\Delta} \cap\left\langle F_{m}\right\rangle$ is a pure $(d-2)$ dimensional subcomplex of $\partial F_{m}$. The barycentric subdivision $\operatorname{sd}(\Delta)$ of $\Delta$ is given by $\operatorname{sd}(\Delta)=\operatorname{sd}(\widetilde{\Delta}) \cup \operatorname{sd}\left(\left\langle F_{m}\right\rangle\right)$ and $\operatorname{sd}(\sigma)=\operatorname{sd}(\widetilde{\Delta}) \cap \operatorname{sd}\left(\left\langle F_{m}\right\rangle\right)$.

We get the following Mayer-Vietoris exact sequence of $A$-modules:

$$
0 \rightarrow k[\operatorname{sd}(\Delta)] \rightarrow k[\operatorname{sd}(\widetilde{\Delta})] \oplus k\left[\operatorname{sd}\left(\left\langle F_{m}\right\rangle\right)\right] \rightarrow k[\operatorname{sd}(\sigma)] \rightarrow 0 .
$$

Here the injection on the left-hand side is given by $\alpha \mapsto(\tilde{\alpha},-\tilde{\alpha})$ and the surjection on the right-hand side by $(\beta, \gamma) \mapsto \tilde{\beta}+\tilde{\gamma}$, where $\tilde{a}$ denotes the obvious projection of $a$ on the appropriate quotient module. (For a subcomplex $\Gamma$ of $\Delta$ and $v \in \Delta_{0} \backslash \Gamma_{0}$, it holds that $x_{v} \cdot k[\Gamma]=0$.)

Let $\Theta=\left\{\theta_{1}, \ldots, \theta_{d}\right\}$ be an l.s.o.p. for $k[\operatorname{sd}(\Delta)], k[\operatorname{sd}(\widetilde{\Delta})]$ and $k\left[\operatorname{sd}\left(F_{m}\right)\right]$, and such that $\left\{\theta_{1}, \ldots, \theta_{d-1}\right\}$ is an l.s.o.p. for $k[\operatorname{sd}(\sigma)]$. This is possible, as the intersection of finitely many non-empty Zariski open sets is non-empty (for $k[\sigma]$, its set of l.s.o.p.'s times $k^{n}$ (for $\theta_{d}$ ) is Zariski open in $k^{d n}$ ). Tensoring the components of the short exact sequence (11) with $A / \Theta$ (over $A$ ), or equivalently, modding out the modules in (1) by $\Theta$, induces the following Tor-long exact sequence:

$$
\begin{aligned}
& \ldots \rightarrow \operatorname{Tor}_{1}(k[\operatorname{sd}(\Delta)], A / \Theta) \rightarrow \operatorname{Tor}_{1}\left(k[\operatorname{sd}(\widetilde{\Delta})] \oplus k\left[\operatorname{sd}\left(\left\langle F_{m}\right\rangle\right)\right], A / \Theta\right) \\
& \rightarrow \operatorname{Tor}_{1}(k[\operatorname{sd}(\sigma)], A / \Theta) \stackrel{\delta}{\rightarrow} \operatorname{Tor}_{0}(k[\operatorname{sd}(\Delta)], A / \Theta) \\
& \rightarrow \operatorname{Tor}_{0}\left(k[\operatorname{sd}(\widetilde{\Delta})] \oplus k\left[\operatorname{sd}\left(\left\langle F_{m}\right\rangle\right)\right], A / \Theta\right) \rightarrow \operatorname{Tor}_{0}(k[\operatorname{sd}(\sigma)], A / \Theta) \rightarrow 0,
\end{aligned}
$$

where $\delta: \operatorname{Tor}_{1}(k[\operatorname{sd}(\sigma)], A / \Theta) \rightarrow \operatorname{Tor}_{0}(k[\operatorname{sd}(\Delta)], A / \Theta)$ is the connecting homomorphism. Below we write $k(\operatorname{sd}(\Delta))$ for $k[\operatorname{sd}(\Delta)] / \Theta$, and similarly $k(\operatorname{sd}(\widetilde{\Delta})), k(\operatorname{sd}(\sigma))$ and $k\left(\operatorname{sd}\left(\left\langle F_{m}\right\rangle\right)\right)$ for $k[\operatorname{sd}(\widetilde{\Delta})] / \Theta, k[\operatorname{sd}(\sigma)] / \Theta$ and $k\left[\operatorname{sd}\left(\left\langle F_{m}\right\rangle\right)\right] / \Theta$ resp.

Using that for $A$-modules $M, N$ and $Q$ it holds that $\operatorname{Tor}_{0}(M, N) \cong M \otimes_{A} N$, $(M \oplus N) \otimes_{A} Q \cong\left(M \otimes_{A} Q\right) \oplus\left(N \otimes_{A} Q\right)$ and that $M / I M \cong M \otimes_{A} A / I$ for an ideal 
$I \triangleleft A$, we get the following exact sequence of $A$-modules:

$$
\operatorname{Tor}_{1}(k[\operatorname{sd}(\sigma)], A / \Theta) \stackrel{\delta}{\rightarrow} k(\operatorname{sd}(\Delta)) \rightarrow k(\operatorname{sd}(\widetilde{\Delta})) \oplus k\left(\operatorname{sd}\left(\left\langle F_{m}\right\rangle\right)\right) \rightarrow k(\operatorname{sd}(\sigma)) \rightarrow 0 .
$$

All the maps in this sequence are grading preserving, where $\operatorname{Tor}_{1}(k[\operatorname{sd}(\sigma)], A / \Theta)$ inherits the grading from (a projective grading preserving resolution of ) sequence (11). From this we deduce the following commutative diagram:

$$
\begin{array}{rlrr}
\operatorname{Tor}_{1}(k[\operatorname{sd}(\sigma)], A / \Theta)_{i} \stackrel{\delta}{\rightarrow} & k(\operatorname{sd}(\Delta))_{i} & \rightarrow & k(\operatorname{sd}(\widetilde{\Delta}))_{i} \oplus k\left(\operatorname{sd}\left(\left\langle F_{m}\right\rangle\right)\right)_{i} \\
& \downarrow \omega^{d-2 i-1} & & \downarrow\left(\omega^{d-2 i-1}, \omega^{d-2 i-1}\right) \\
& k(\operatorname{sd}(\Delta))_{d-1-i} & \rightarrow & k(\operatorname{sd}(\widetilde{\Delta}))_{d-1-i} \oplus k\left(\operatorname{sd}\left(\left\langle F_{m}\right\rangle\right)\right)_{d-1-i}
\end{array}
$$

where $\omega$ is a degree one element in $A$. Since $F_{m}$ is a $(d-1)$-simplex we know from the base of the induction that multiplication

$$
\omega^{d-2 i-1}: k\left(\operatorname{sd}\left(\left\langle F_{m}\right\rangle\right)\right)_{i} \rightarrow k\left(\operatorname{sd}\left(\left\langle F_{m}\right\rangle\right)\right)_{d-1-i}
$$

is an injection for a generic choice of $\omega$ in $A_{1}$. (Note that if $G$ is a Zariski open set in $k\left[x_{v} \mid v \in\left(F_{m}\right)_{0}\right]_{1}$, then $G \times k\left[x_{v} \mid v \in \Delta_{0} \backslash\left(F_{m}\right)_{0}\right]_{1}$ is Zariski open in $A_{1}$.)

By construction, $\widetilde{\Delta}$ is shellable and therefore by the induction hypothesis the multiplication

$$
\omega^{d-2 i-1}: \quad k(\operatorname{sd}(\widetilde{\Delta}))_{i} \rightarrow k(\operatorname{sd}(\widetilde{\Delta}))_{d-1-i}
$$

is an injection for generic $\omega$. Since the intersection of two non-empty Zariski open sets is non-empty, the multiplication

$\left(\omega^{d-2 i-1}, \omega^{d-2 i-1}\right): k(\operatorname{sd}(\widetilde{\Delta}))_{i} \oplus k\left(\operatorname{sd}\left(\left\langle F_{m}\right\rangle\right)\right)_{i} \rightarrow k(\operatorname{sd}(\widetilde{\Delta}))_{d-1-i} \oplus k\left(\operatorname{sd}\left(\left\langle F_{m}\right\rangle\right)\right)_{d-1-i}$ is an injection for a generic $\omega \in A_{1}$.

Our aim is to show that $\operatorname{Tor}_{1}(k[\operatorname{sd}(\sigma)], A / \Theta)_{i}=0$ for $0 \leq i \leq\left\lfloor\frac{d-2}{2}\right\rfloor$. As soon as this is shown, the above commutative diagram implies that the multiplication

$$
\omega^{d-2 i-1}: k(\operatorname{sd}(\Delta))_{i} \rightarrow k(\operatorname{sd}(\Delta))_{d-1-i}
$$

is injective for $0 \leq i \leq\left\lfloor\frac{d-2}{2}\right\rfloor$ and $\omega$ as above.

For the computation of $\operatorname{Tor}_{1}(k[\operatorname{sd}(\sigma)], A / \Theta)$ we consider the following exact sequence of $A$-modules:

$$
0 \rightarrow \Theta A \rightarrow A \rightarrow A / \Theta \rightarrow 0
$$

Since $\operatorname{Tor}_{0}(M, N) \cong M \otimes_{A} N$ and $\operatorname{Tor}_{1}(A, M)=0$ for the $A$-modules $M$ and $N$, we get the following Tor-long exact sequence:

$$
0 \rightarrow \operatorname{Tor}_{1}(A / \Theta, k[\operatorname{sd}(\sigma)]) \rightarrow \Theta A \otimes_{A} k[\operatorname{sd}(\sigma)] \rightarrow k[\operatorname{sd}(\sigma)] \rightarrow A / \Theta \otimes_{A} k[\operatorname{sd}(\sigma)] \rightarrow 0 .
$$

From the exactness of this sequence we deduce that $\operatorname{Tor}_{1}(A / \Theta, k[\operatorname{sd}(\sigma)])=$ $\operatorname{Ker}\left(\Theta A \otimes_{A} k[\operatorname{sd}(\sigma)] \rightarrow k[\operatorname{sd}(\sigma)]\right)$. Since we have $\operatorname{Tor}_{1}(k[\operatorname{sd}(\sigma)], A / \Theta) \cong$ $\operatorname{Tor}_{1}(A / \Theta, k[\operatorname{sd}(\sigma)])$, and by the fact that the isomorphism is degree preserving, we finally get that $\operatorname{Tor}_{1}(k[\operatorname{sd}(\sigma)], A / \Theta) \cong \operatorname{Ker}\left(\Theta A \otimes_{A} k[\operatorname{sd}(\sigma)] \rightarrow k[\operatorname{sd}(\sigma)]\right)$ as graded $A$-modules. The grading of $\Theta A \otimes_{A} k[\operatorname{sd}(\sigma)]$ is given by $\operatorname{deg}\left(f \otimes_{A} g\right)=$ $\operatorname{deg}_{A}(f)+\operatorname{deg}_{A}(g)$, where $\operatorname{deg}_{A}$ refers to the grading induced by $A$.

As mentioned before, for generic $\Theta, \widetilde{\Theta}:=\left\{\theta_{1}, \ldots, \theta_{d-1}\right\}$ is an l.s.o.p. for $k[\operatorname{sd}(\sigma)]$, and hence a regular sequence for $k[\operatorname{sd}(\sigma)]$ due to the Cohen-Macaulayness of $\sigma$. Thus the kernel of the map

$$
\left(\Theta A \otimes_{A} k[\operatorname{sd}(\sigma)]\right)_{i} \rightarrow(k[\operatorname{sd}(\sigma)])_{i} ; \quad b \otimes f \mapsto b f
$$


is zero iff the kernel of the map

$$
\left(\left(\theta_{d}\right) \otimes_{A}(k[\operatorname{sd}(\sigma)] / \widetilde{\Theta})\right)_{i} \rightarrow(k[\operatorname{sd}(\sigma)] / \widetilde{\Theta})_{i} ; \quad \theta_{d} \otimes f \mapsto \theta_{d} f
$$

is zero, which is the case iff the kernel of the multiplication map

$$
\theta_{d}^{j}:(k[\operatorname{sd}(\sigma)] / \widetilde{\Theta})_{i-j} \rightarrow(k[\operatorname{sd}(\sigma)] / \widetilde{\Theta})_{i} ; f \mapsto \theta_{d}^{j} f
$$

is zero for every $1 \leq j \leq i$. (We have a shift of $(-j)$ in the grading since the last map $\theta_{d}^{j}$ increases the degree by $+j$.)

By construction, $\sigma$ is a pure subcomplex of the boundary of a $(d-1)$-simplex and thus is shellable. Since $\operatorname{dim}(\sigma)=d-2$ the induction hypothesis applies to $\operatorname{sd}(\sigma)$. Thus, the multiplication

$$
\theta_{d}^{d-2 l-2}: \quad(k[\operatorname{sd}(\sigma)] / \widetilde{\Theta})_{l} \rightarrow(k[\operatorname{sd}(\sigma)] / \widetilde{\Theta})_{d-l-2}
$$

is an injection for $0 \leq l \leq\left\lfloor\frac{d-3}{2}\right\rfloor$ for a generic degree one element $\theta_{d}$. In particular, the multiplication

$$
\theta_{d}^{j}:(k[\operatorname{sd}(\sigma)] / \widetilde{\Theta})_{i-j} \rightarrow(k[\operatorname{sd}(\sigma)] / \widetilde{\Theta})_{i}
$$

is injective for $1 \leq j \leq i \leq\left\lfloor\frac{d-3}{2}\right\rfloor+1=\left\lfloor\frac{d-1}{2}\right\rfloor$. Thus, $\operatorname{Tor}_{1}(k[\operatorname{sd}(\sigma)], A / \Theta)_{i}=0$ for $1 \leq i \leq\left\lfloor\frac{d-1}{2}\right\rfloor$. In particular, $\operatorname{Tor}_{1}(k[\operatorname{sd}(\sigma)], A / \Theta)_{i}=0$ for $1 \leq i \leq\left\lfloor\frac{d-2}{2}\right\rfloor$. Note that $\left(\Theta A \otimes_{A} k[\operatorname{sd}(\sigma)]\right)_{0}=0$; hence $\operatorname{Tor}_{1}(k[\operatorname{sd}(\sigma)], A / \Theta)_{0}=0$. To summarize, $\operatorname{Tor}_{1}(k[\operatorname{sd}(\sigma)], A / \Theta)_{i}=0$ for $0 \leq i \leq\left\lfloor\frac{d-2}{2}\right\rfloor$, which completes the proof.

\section{Combinatorial Consequences}

We are now going to discuss some combinatorial consequences of Theorem 1.1

For a $(d-1)$-dimensional simplicial complex $\Delta$ let its $g$-vector be $g^{\Delta}:=\left(g_{0}^{\Delta}, g_{1}^{\Delta}\right.$, $\left.\ldots, g_{\left\lfloor\frac{d}{2}\right\rfloor}^{\Delta}\right)$, where $g_{0}^{\Delta}=1$ and $g_{i}^{\Delta}=h_{i}^{\Delta}-h_{i-1}^{\Delta}$ for $1 \leq i \leq\left\lfloor\frac{d}{2}\right\rfloor$. A sequence $\left(a_{0}, \ldots, a_{t}\right)$ is called an $M$-sequence if it is the Hilbert function of a standard graded Artinian $k$-algebra. Macaulay [7] gave a characterization of such sequences by means of numerical conditions on their elements (see e.g. 14]).

Recall that shellable complexes are CM (e.g. 4]). While the converse is not true, Stanley showed that these two families of complexes have the same set of $h$-vectors:

Theorem 3.1 (Theorem II.3.3 [14]). Let $s=\left(h_{0}, \ldots, h_{d}\right)$ be a sequence of integers. The following conditions are equivalent:

(i) $s$ is the h-vector of a shellable simplicial complex.

(ii) $s$ is the h-vector of a Cohen-Macaulay simplicial complex.

(iii) $s$ is an $M$-sequence.

We are now going to prove some $f$-vector corollaries of Theorem 1.1

Proof of Corollary 1.3. For any simplicial complex $\Gamma, h^{\mathrm{sd}(\Gamma)}$ is a function of $h^{\Gamma}$. (For an explicit formula, see Theorem 4.2 below, obtained in 3 .) Hence, together with Theorem 3.1 we can assume that $\Delta$ is shellable. Let $\operatorname{dim} \Delta=d-1$.

By Theorem 1.1] for a generic l.s.o.p. $\Theta$ and a generic degree one element $\omega$, the multiplication

$$
\omega^{d-1-2 i}: \quad(k[\operatorname{sd}(\Delta)] / \Theta)_{i} \rightarrow(k[\operatorname{sd}(\Delta)] / \Theta)_{d-1-i}
$$

is an injection for $0 \leq i \leq\left\lfloor\frac{d-2}{2}\right\rfloor$; hence

$$
\omega:(k[\operatorname{sd}(\Delta)] / \Theta)_{i} \rightarrow(k[\operatorname{sd}(\Delta)] / \Theta)_{i+1}
$$


is an injection as well. (This conclusion is vacuous for $d \leq 1$.) Therefore, as Cohen-Macaulayness implies $h_{i}^{\mathrm{sd}(\Delta)}=\operatorname{dim}_{k}(k[\operatorname{sd}(\Delta)] / \Theta)_{i}$, we get that $g_{i}^{\operatorname{sd}(\Delta)}=$ $\operatorname{dim}_{k}(k[\operatorname{sd}(\Delta)] /(\Theta, \omega))_{i}$ for $0 \leq i \leq\left\lfloor\frac{d}{2}\right\rfloor$. Hence $g^{\operatorname{sd}(\Delta)}$ is an $M$-sequence.

Corollary 3.2. Let $\Delta$ be a $(d-1)$-dimensional Cohen-Macaulay simplicial complex. Then $h_{d-i-1}^{\mathrm{sd}(\Delta)} \geq h_{i}^{\mathrm{sd}(\Delta)}$ for any $0 \leq i \leq\left\lfloor\frac{d-2}{2}\right\rfloor$.

Proof. Again, by Theorems 4.2 and 3.1 we can assume that $\Delta$ is shellable. By Theorem 1.1, for a generic l.s.o.p. $\Theta$ of $k[\operatorname{sd}(\Delta)]$, the multiplication

$$
\omega^{d-1-2 i}:(k[\operatorname{sd}(\Delta)] / \Theta)_{i} \rightarrow(k[\operatorname{sd}(\Delta)] / \Theta)_{d-1-i}
$$

is an injection for $1 \leq i \leq\left\lfloor\frac{d-2}{2}\right\rfloor$ and a generic degree one element $\omega$. Since $h_{i}^{\mathrm{sd}(\Delta)}=\operatorname{dim}_{k}(k[\operatorname{sd}(\Delta)] / \Theta)_{i}$ this implies $h_{i}^{\mathrm{sd}(\Delta)} \leq h_{d-1-i}^{\mathrm{sd}(\Delta)}$.

Next we verify the almost strong Lefschetz property for polytopal complexes. The proof essentially follows the same steps as the one of Theorem 1.1] we will indicate the differences.

A polytopal complex is a finite, non-empty collection $\mathcal{C}$ of polytopes (called the faces of $\mathcal{C}$ ) in some $\mathbb{R}^{t}$ that contains all the faces of its polytopes, and such that the intersection of two of its polytopes is a face of each of them. Notions such as facets, dimension, pureness and barycentric subdivision are defined as usual.

Shellability extends to polytopal complexes as follows (see, e.g., [16] for more details).

Definition 3.3. Let $\mathcal{C}$ be a pure $(d-1)$-dimensional polytopal complex. A shelling of $\mathcal{C}$ is a linear ordering $F_{1}, F_{2}, \ldots, F_{m}$ of the facets of $\mathcal{C}$ such that either $\mathcal{C}$ is 0 dimensional, or it satisfies the following conditions:

(i) The boundary complex $\mathcal{C}\left(\partial F_{1}\right)$ of the first facet $F_{1}$ has a shelling.

(ii) For $1<j \leq m$ the intersection of the facet $F_{j}$ with the previous facets is non-empty and is the beginning segment of a shelling of the $(d-2)$ dimensional boundary complex of $F_{j}$; that is,

$$
F_{j} \cap\left(\bigcup_{i=1}^{j-1} F_{i}\right)=G_{1} \cup G_{2} \cup \ldots \cup G_{r}
$$

for some shelling $G_{1}, G_{2}, \ldots, G_{r}, \ldots, G_{t}$ of $\mathcal{C}\left(\partial F_{j}\right)$, and $1 \leq r \leq t$.

A polytopal complex is called shellable if it is pure and has a shelling.

Theorem 3.4. Let $\Delta$ be a shellable $(d-1)$-dimensional polytopal complex. Then $\operatorname{sd}(\Delta)$ is almost strong Lefschetz over $\mathbb{R}$. In particular, $g^{\operatorname{sd}(\Delta)}$ is an $M$-sequence.

Proof. We give a sketch of the proof, indicating the needed modifications in the proof of Theorem 1.1.

We use induction on the dimension and the number of facets $f_{d-1}^{\Delta}$ of $\Delta$. For $f_{d-1}^{\Delta}=1$, note that the barycentric subdivision of a polytope is combinatorially isomorphic to a cone over the boundary of a simplicial polytope (see [5]).

Theorem 2.2 implies that $\operatorname{sd}(\partial P)$ is $(d-1)$-Lefschetz over $\mathbb{R}$. By Lemma 2.1 the same holds for cone $(\operatorname{sd}(\partial P))=\operatorname{sd}(P)$. Together with the $\operatorname{dim} \Delta=0$ case, this provides the base of induction.

The induction step works as in the proof of Theorem 1.1 . 
Note that in the above proof we really need the classical $g$-theorem, whereas in the proof of Theorem 1.1 it was not required.

\section{New inequalities For the ReFined Eulerian STAtistics ON PERMUTATIONS}

In [3, Brenti and Welker give a precise description of the $h$-vector of the barycentric subdivision of a simplicial complex in terms of the $h$-vector of the original complex. The coefficients that occur in this representation are a refinement of the Eulerian statistics on permutations.

Let $S_{d}$ denote the symmetric group on $[d]$, and let $\sigma \in S_{d}$. We write $D(\sigma):=$ $\{i \in[d-1] \mid \sigma(i)>\sigma(i+1)\}$ for the descent set of $\sigma$ and $\operatorname{des}(\sigma):=\# D(\sigma)$ counts the number of descents of $\sigma$. For $0 \leq i \leq d-1$ and $1 \leq j \leq d$ we set $A(d, i, j):=\#\left\{\sigma \in S_{d} \mid \operatorname{des}(\sigma)=i, \sigma(1)=j\right\}$. Brenti and Welker showed that these numbers satisfy the following symmetry:

Lemma 4.1 ([3, Lemma 2.5]).

$$
A(d, i, j)=A(d, d-1-i, d+1-j)
$$

for $d \geq 1,1 \leq j \leq d$ and $0 \leq i \leq d-1$.

The following theorem establishes the relation between the $h$-vector of a simplicial complex and the $h$-vector of its barycentric subdivision. As stated in [3] the result actually holds in the generality of Boolean cell complexes, also known as simplicial posets.

Theorem 4.2 ([3, Theorem 2.2]). Let $\Delta$ be a (d-1)-dimensional simplicial complex and let $\operatorname{sd}(\Delta)$ be its barycentric subdivision. Then

$$
h_{j}^{\mathrm{sd}(\Delta)}=\sum_{r=0}^{d} A(d+1, j, r+1) h_{r}^{\Delta}
$$

for $0 \leq j \leq d$.

In order to prove some new inequalities for the $A(d, i, j)$ 's we will need the following characterization of the $h$-vector of a shellable simplicial complex due to McMullen and Walkup.

Proposition 4.3 ([4, Corollary 5.1.14]). Let $\Delta$ be a shellable $(d-1)$-dimensional simplicial complex with shelling $F_{1}, \ldots, F_{m}$. For $2 \leq j \leq m$, let $r_{j}$ be the number of facets of $\left\langle F_{j}\right\rangle \cap\left\langle F_{1}, \ldots, F_{j-1}\right\rangle$ and set $r_{1}=0$. Then $h_{i}^{\Delta}=\#\left\{j \mid r_{j}=i\right\}$ for $i=0, \ldots, d$. In particular, the numbers $h_{j}^{\Delta}$ do not depend on the particular shelling.

It is easily seen that $r_{j}=\# \operatorname{res}\left(F_{j}\right)$. We will use this fact in the proof of the following corollary.

Corollary 4.4. (i) $A(d+1, j, r+1) \leq A(d+1, d-1-j, r+1)$ for $d \geq 0$, (ii) $0 \leq r \leq d$ and $0 \leq j \leq\left\lfloor\frac{d-2}{2}\right\rfloor$.

$$
\begin{aligned}
& A(d+1,0, r+1) \leq A(d+1,1, r+1) \leq \ldots \leq A\left(d+1,\left\lfloor\frac{d}{2}\right\rfloor, r+1\right) \\
& \text { and } \\
& A(d+1, d, r+1) \leq A(d+1, d-1, r+1) \leq \ldots \leq A\left(d+1,\left\lceil\frac{d}{2}\right\rceil, r+1\right)
\end{aligned}
$$


for $d \geq 1$ and $0 \leq r \leq d$. (For $d$ odd, $A\left(d+1,\left\lfloor\frac{d}{2}\right\rfloor, r+1\right)$ may be larger or smaller than $A\left(d+1,\left\lceil\frac{d}{2}\right\rceil, r+1\right)$.)

Proof. Let $\Delta$ be a shellable $(d-1)$-dimensional simplicial complex. Let $F_{1}, \ldots, F_{m}$ be a shelling of $\Delta$ with $m \geq 2$ and set $\widetilde{\Delta}:=\left\langle F_{1}, \ldots, F_{m-1}\right\rangle$. Since $\operatorname{sd}(\widetilde{\Delta})$ is a subcomplex of $\operatorname{sd}(\Delta)$ we get the following short exact sequence of $A$-modules for $A=k\left[x_{1}, \ldots, x_{f_{0}^{\mathrm{sd}(\Delta)}}\right]:$

$$
0 \rightarrow I \rightarrow k[\operatorname{sd}(\Delta)] \rightarrow k[\operatorname{sd}(\widetilde{\Delta})] \rightarrow 0,
$$

where $I$ denotes the kernel of the projection on the right-hand side. Let $\Theta$ be an l.s.o.p. for both $k[\operatorname{sd}(\Delta)]$ and $k[\operatorname{sd}(\widetilde{\Delta})]$. As $\widetilde{\Delta}$ is shellable it is $\mathrm{CM}$ and therefore $\operatorname{sd}(\widetilde{\Delta})$ is $\mathrm{CM}$ as well. Hence dividing out by $\Theta$ yields the following exact sequence of $A$-modules:

$$
0 \rightarrow I /(I \cap \Theta) \rightarrow k[\operatorname{sd}(\Delta)] / \Theta \rightarrow k[\operatorname{sd}(\widetilde{\Delta})] / \Theta \rightarrow 0 .
$$

Consider the following commutative diagram:

$$
\begin{array}{cccc}
0 \rightarrow & I /(I \cap \Theta)_{i} & \rightarrow & (k[\operatorname{sd}(\Delta)] / \Theta)_{i} \\
& \downarrow \omega^{d-1-2 i} & & \downarrow \omega^{d-1-2 i} \\
0 & \rightarrow \quad I /(I \cap \Theta)_{d-1-i} & \rightarrow & (k[\operatorname{sd}(\Delta)] / \Theta)_{d-1-i}
\end{array}
$$

where $\omega$ is in $A_{1}$. By Theorem 1.1 multiplication,

$$
\omega^{d-2 i-1}:(k[\operatorname{sd}(\Delta)] / \Theta)_{i} \rightarrow(k[\operatorname{sd}(\Delta)])_{d-1-i}
$$

is an injection for $0 \leq i \leq\left\lfloor\frac{d-2}{2}\right\rfloor$ and generic $\omega$. It hence follows that also the multiplication

$$
\omega^{d-1-2 i}: \quad(I /(I \cap \Theta))_{i} \rightarrow(I /(I \cap \Theta))_{d-1-i}
$$

is an injection for $0 \leq i \leq\left\lfloor\frac{d-2}{2}\right\rfloor$.

Furthermore, we deduce from the sequence (2) that $\operatorname{dim}_{k}(I /(I \cap \Theta))_{t}=h_{t}^{\operatorname{sd}(\Delta)}-$ $h_{t}^{\operatorname{sd}(\widetilde{\Delta})}$ for $0 \leq t \leq d$.

In order to compute this difference we determine the change in the $h$-vector of $\widetilde{\Delta}$ when adding the last facet $F_{m}$ of the shelling. Let $r_{m}:=\# \operatorname{res}\left(F_{m}\right)$. Proposition 4.3 implies $h_{r_{m}}^{\Delta}=h_{r_{m}}^{\widetilde{\Delta}}+1$ and $h_{i}^{\Delta}=h_{i}^{\widetilde{\Delta}}$ for $i \neq r_{m}$. Using Theorem 4.2 we deduce:

$$
\begin{aligned}
h_{i}^{\operatorname{sd}(\Delta)} & =\sum_{r=0}^{d} A(d+1, i, r+1) h_{r}^{\Delta} \\
& =\sum_{r=0}^{d} A(d+1, i, r+1) h_{r}^{\widetilde{\Delta}}+A\left(d+1, i, r_{m}+1\right) \\
& =h_{i}^{\operatorname{sd}(\widetilde{\Delta})}+A\left(d+1, i, r_{m}+1\right) .
\end{aligned}
$$

Thus $\operatorname{dim}_{k}(I /(I \cap \Theta))_{i}=A\left(d+1, i, r_{m}+1\right)$ for $0 \leq i \leq\left\lfloor\frac{d-2}{2}\right\rfloor$. From (3) it follows that $A\left(d+1, i, r_{m}+1\right) \leq A\left(d+1, d-1-i, r_{m}+1\right)$.

Take $\Delta$ to be the boundary of the $d$-simplex. Since in this case $h_{i}^{\Delta}=1$ for $0 \leq i \leq d$, restriction faces of all possible sizes occur in a shelling of $\Delta$, and it follows that $A(d+1, i, r+1) \leq A(d+1, d-1-i, r+1)$ for every $0 \leq r \leq d$ and $0 \leq i \leq\left\lfloor\frac{d-2}{2}\right\rfloor$. This shows (i). 
To show (ii) we use that the injections in (3) induce injections

$$
\omega: \quad(I /(I \cap \Theta))_{i} \rightarrow(I /(I \cap \Theta))_{i+1}
$$

for $0 \leq i \leq\left\lfloor\frac{d-2}{2}\right\rfloor$. Thus, $A\left(d+1, i, r_{m}+1\right) \leq A\left(d+1, i+1, r_{m}+1\right)$. The same reasoning as in (i) shows that $A(d+1, i, r+1) \leq A(d+1, i+1, r+1)$ for $0 \leq i \leq\left\lfloor\frac{d-2}{2}\right\rfloor$ and $0 \leq r \leq d$. The second part of (ii) follows from the first part using Lemma 4.1 .

Example 4.5. $A(6,2,3)=60>48=A(6,3,3)$ while $A(6,2,4)=48<60=$ $A(6,3,4)$. This shows that for $d$ odd $A\left(d+1,\left\lfloor\frac{d}{2}\right\rfloor, r+1\right)$ may be larger or smaller than $A\left(d+1,\left\lceil\frac{d}{2}\right\rceil, r+1\right)$.

Recall that a sequence of integers $s=\left(s_{0}, \ldots, s_{d}\right)$ is called unimodal if there is a $0 \leq j \leq d$ such that $s_{0} \leq \ldots \leq s_{j} \geq \ldots \geq s_{d}$. We call $s_{j}$ a peak of this sequence and say that it is at position $j$ (note that $j$ may not be unique).

Remark 4.6. From [3] it can already be deduced that the sequence $(A(d+1,0$, $r+1), \ldots, A(d+1, d, r+1))$ is unimodal. Applying the linear transformation of Theorem 4.2 to the $(r+1)$ st unit vector yields the sequence $(A(d+1,0, r+1), \ldots$, $A(d+1, d, r+1))$. It then follows from [3, Theorem 3.1, Remark 3.3] that the generating polynomial of this sequence is real-rooted. Since $A(d, i, r+1) \geq 1$ for $i \geq$ 1 the sequence $(A(d+1,0, r+1), \ldots, A(d+1, d, r+1))$ has no internal zeros. Together with the real-rootedness this implies that $(A(d+1,0, r+1), \ldots, A(d+1, d, r+1))$ is unimodal. However, this argument tells us nothing about the position of the peak.

Recall that a regular CW-complex $\Delta$ is called a Boolean cell complex if for each $A \in \Delta$ the lower interval $[\emptyset, A]:=\left\{B \in \Delta \mid \emptyset \leq_{\Delta} B \leq_{\Delta} A\right\}$ is a Boolean lattice, where $A \leq_{\Delta} A^{\prime}$ if $A$ is contained in the closure of $A^{\prime}$ for $A, A^{\prime} \in \Delta$. In [3] it was shown that the $h$-vector of the barycentric subdivision of a Boolean cell complex with non-negative $h$-entries is unimodal. What remains open is the location of its peak. Using Corollary 4.4 we can solve this problem:

Corollary 4.7. Let $\Delta$ be a $(d-1)$-dimensional Boolean cell complex with $h_{i}^{\Delta} \geq 0$ for $0 \leq i \leq d$. Then $h^{\operatorname{sd}(\Delta)}$ has a peak at position $\frac{d}{2}$ if $d$ is even and at position $\frac{\bar{d}-1}{2}$ or $\frac{d+1}{2}$ if $d$ is odd. In particular, this assertion holds for CM simplicial complexes.

Proof. Since $h_{i}^{\Delta} \geq 0$ for $0 \leq i \leq d$, by Theorem 4.2 and Corollary 4.4 (ii), we deduce

$$
\begin{array}{rll}
h_{j}^{\mathrm{sd}(\Delta)} & = & \sum_{r=0}^{d} A(d+1, j, r+1) h_{r}^{\Delta} \\
\text { Corollary } 4.4(\text { ii }) & \sum_{r=0}^{d} A(d+1, j+1, r+1) h_{r}^{\Delta}=h_{j+1}^{\mathrm{sd}(\Delta)}
\end{array}
$$

for $0 \leq j \leq\left\lfloor\frac{d-2}{2}\right\rfloor$. Thus $h_{0}^{\operatorname{sd}(\Delta)} \leq h_{1}^{\operatorname{sd}(\Delta)} \leq \ldots \leq h_{\left\lfloor\frac{d}{2}\right\rfloor}^{\operatorname{sd}(\Delta)}$.

Similarly one shows $h_{\left\lceil\frac{d}{2}\right\rceil}^{\mathrm{sd}(\Delta)} \geq h_{\left\lceil\frac{d}{2}\right\rceil+1}^{\mathrm{sd}(\Delta)} \geq \ldots \geq h_{d}^{\mathrm{sd}(\Delta)}$, when applying Corollary 4.4 (ii) for $j \geq\left\lceil\frac{d}{2}\right\rceil$.

If $d$ is even, then $\left\lfloor\frac{d}{2}\right\rfloor=\left\lceil\frac{d}{2}\right\rceil=\frac{d}{2}$ and $h^{\mathrm{sd}(\Delta)}$ has a peak at position $\frac{d}{2}$. 
Remark 4.8. For non-empty $\Delta$ as above, strict inequalities for $h^{\mathrm{sd}(\Delta)}$ can be shown as follows:

$$
h_{0}^{\operatorname{sd}(\Delta)}<h_{1}^{\operatorname{sd}(\Delta)}<\ldots<h_{\left\lfloor\frac{d-1}{2}\right\rfloor}^{\operatorname{sd}(\Delta)} \text { and } h_{\left\lceil\frac{d}{2}\right\rceil}^{\operatorname{sd}(\Delta)}>\ldots>h_{d}^{\operatorname{sd}(\Delta)} .
$$

Proof. $A(d+1, j, 1)=E(d, j)$, where $E(d, j)$ is the Eulerian number equal to the number of permutations on $[d]$ with $j$ descents. It is well known that the Euler polynomial $\sum_{0 \leq j \leq d-1} E(d, j) t^{j}$ has a non-negative integer expansion w.r.t. the independent set of polynomials $\left\{t^{i}(t+1)^{d-1-2 i}\right\}_{0 \leq i \leq\left\lfloor\frac{d-1}{2}\right\rfloor}$; see [6], or the later [12] for a combinatorial proof. Note that the coefficient of $(t+1)^{d-1}$ is 1 for $d \geq 1$. Thus, the fact that for any non-negative integer $k$ the binomial coefficients $\left(\begin{array}{l}k \\ l\end{array}\right)$ strictly increase for $0 \leq l \leq\left\lfloor\frac{k}{2}\right\rfloor$ and strictly decrease for $\left\lceil\frac{k}{2}\right\rceil \leq l \leq k$ implies that the $A(d+1, j, 1)$ s strictly increase for $0 \leq j \leq\left\lfloor\frac{d-1}{2}\right\rfloor$ and strictly decrease for $\left\lceil\frac{d-1}{2}\right\rceil \leq j \leq d$ (note that $\left.A(d+1, d-1,1)=1>0=A(d+1, d, 1)\right)$. As $\Delta$ is non-empty, $h_{0}^{\Delta}=1$. Looking at the proof of Corollary 4.7, comparing the $r=0$ summands on both sides of the inequality implies the desired strict inequalities.

Example 4.9. If $d$ is odd, depending on whether $h_{\left\lfloor\frac{d}{2}\right\rfloor}^{\operatorname{sd}(\Delta)} \leq h_{\left\lceil\frac{d}{2}\right\rceil}^{\operatorname{sd}(\Delta)}$ or vice versa the peak of $h^{\operatorname{sd}(\Delta)}$ is at position $\frac{d-1}{2}$ or $\frac{d+1}{2}$. For example, for $d=3$ let $\Delta$ be the 2 -skeleton of the 4 -simplex. Then $h^{\Delta}=(1,2,3,4)$ and $h^{\text {sd( }(\Delta)}=(1,22,33,4)$, i.e. the peak is at position $\frac{3+1}{2}=2$.

If $\Delta$ consists of 2 triangles intersecting along one edge, i.e. $\Delta:=\langle\{1,2,3\},\{2,3,4\}\rangle$, then $h^{\Delta}=(1,1,0,0)$ and $h^{\operatorname{sd}(\Delta)}=(1,8,3,0)$. In this case the $h$-vector peaks at position $\frac{3-1}{2}=1$.

If $d$ is even, taking $\Delta$ to be a $(d-1)$-dimensional simplex shows that $h_{\frac{d}{2}-1}^{\mathrm{sd}(\Delta)}=$ $h_{\frac{d}{2}}^{\mathrm{sd}(\Delta)}$ may occur.

Using Corollary 4.4 we also establish the following inequalities, compactly summarized later in Corollary 4.11.

Corollary 4.10. (i) $A(d+1, j, 1) \leq A(d+1, j, 2) \leq \ldots \leq A(d+1, j, d+1)$ for $\left\lceil\frac{d+1}{2}\right\rceil=\left\lfloor\frac{d+2}{2}\right\rfloor \leq j \leq d$.

(ii) $A(d+1, j, 1) \geq A(d+1, j, 2) \geq \ldots \geq A(d+1, j, d+1)$ for $0 \leq j \leq\left\lfloor\frac{d-1}{2}\right\rfloor$.

(iii) $A\left(d+1, \frac{d}{2}, 1\right) \leq A\left(d+1, \frac{d}{2}, 2\right) \leq \ldots \leq A\left(d+1, \frac{d}{2}, \frac{d}{2}+1\right) \geq A\left(d+1, \frac{d}{2}, \frac{d}{2}+2\right) \geq$ $\ldots \geq A\left(d+1, \frac{d}{2}, d+1\right)$ if $d$ is even.

(iv) $A(d+1, j, 1)=A(d+1, j+1, d+1)$ for $0 \leq j \leq d-1$.

Proof. To prove (i) we need to show that $A(d+1, j, r) \leq A(d+1, j, r+1)$ for $1 \leq r \leq d$ and $\left\lfloor\frac{d+2}{2}\right\rfloor \leq j \leq d$. For $j=d$ this follows from $\left\{\sigma \in S_{d+1} \mid \operatorname{des}(\sigma)=\right.$ $d\}=\{(d+1) d \ldots 21\}$. Let $C_{j, r}^{d}:=\left\{\sigma \in S_{d+1} \mid \operatorname{des}(\sigma)=j, \sigma(1)=r\right\}$. Consider the following map:

$$
\begin{array}{ccc}
\phi_{j, r}^{d}:\left\{\sigma \in C_{j, r}^{d} \mid \sigma(2) \neq r+1\right\} & \rightarrow & \left\{\sigma \in C_{\sigma, r+1}^{d} \mid \sigma(2) \neq r\right\} \\
& \mapsto & (r, r+1) \sigma .
\end{array}
$$

For $\sigma \in C_{j, r}^{d}$, if $\operatorname{des}(\sigma)=j$ and $\sigma(2) \neq r+1$, then $\sigma$ and $(r, r+1) \sigma$ have the same descent set; hence $\operatorname{des}((r, r+1) \sigma)=j$ as well.

As $((r, r+1) \sigma)(1)=r+1$ the function $\phi_{j, r}^{d}$ is well-defined. Since $(r, r+1)^{2}=\mathrm{id}$ it follows that $\phi_{j, r}^{d}$ is invertible and therefore $\#\left\{\sigma \in C_{j, r}^{d} \mid \sigma(2) \neq r+1\right\}=\#\{\sigma \in$ $\left.C_{j, r+1}^{d} \mid \sigma(2) \neq r\right\}$. 
If $\sigma \in C_{j, r}^{d}$ and $\sigma(2)=r+1$, then all of the $j$ descents must occur at position at least 2 .

The sequence $\tilde{\sigma}=(r+1) \sigma(3) \ldots \sigma(d+1)$ can be identified with a permutation $\tau$ in $S_{d}$ with $\tau(1)=r$ and vice versa via the order preserving map $[d+1] \backslash\{r\} \rightarrow[d]$; hence the descent set is preserved under this identification. Therefore $\#\left\{\sigma \in C_{j, r}^{d} \mid \sigma(2)=\right.$ $r+1\}=\#\left\{\sigma \in C_{j, r}^{d-1}\right\}=A(d, j, r)$. On the other hand, if $\sigma \in C_{j, r+1}^{d}$ and $\sigma(2)=r$, then $\sigma$ has exactly $j-1$ descents at positions $\{2, \ldots, d\}$. A similar argument then implies $\#\left\{\sigma \in C_{j, r+1}^{d} \mid \sigma(2)=r\right\}=\#\left\{\sigma \in C_{j-1, r}^{d-1}\right\}=A(d, j-1, r)$.

By Corollary 4.4(ii) it holds that $A(d, j, r) \leq A(d, j-1, r)$ for $d-2 \geq j-$ $1 \geq\left\lceil\frac{d-1}{2}\right\rceil$, i.e. $d-1 \geq j \geq\left\lceil\frac{d+1}{2}\right\rceil=\left\lfloor\frac{d+2}{2}\right\rfloor$. Combining the above, we obtain $A(d+1, j, r) \leq A(d+1, j, r+1)$ for $1 \leq r \leq d$ and $\left\lfloor\frac{d+2}{2}\right\rfloor \leq j \leq d-1$, and (i) follows.

(ii) follows directly from (i) and Lemma 4.1

For the proof of (iii) we only show $A\left(d+1, \frac{d}{2}, 1\right) \leq A\left(d+1, \frac{d}{2}, 2\right) \leq \ldots \leq$ $A\left(d+1, \frac{d}{2}, \frac{d}{2}+1\right)$. The other inequalities in (iii) follow directly from this part by Lemma 4.1. The proof of (i) shows that $\#\left\{\sigma \in C_{\frac{d}{2}, r}^{d} \mid \sigma(2) \neq r+1\right\}=\#\{\sigma \in$ $\left.C_{\frac{d}{2}, r+1}^{d} \mid \sigma(2) \neq r\right\}$. As in the proof of (i), it remains to prove that $A\left(d, \frac{d}{2}, r\right) \leq$ $A\left(d, \frac{d}{2}-1, r\right)$ for $1 \leq r \leq \frac{d}{2}$. By Lemma 4.1 it holds that $A\left(d, \frac{d}{2}, r\right)=A\left(d, \frac{d}{2}-1, d+\right.$ $1-r)$. For $1 \leq r \leq \frac{d}{2}$ we have $r \leq d+1-r$ and (ii) then implies $A\left(d, \frac{d}{2}-1, r\right) \geq$ $A\left(d, \frac{d}{2}-1, d+1-r\right)$ which finishes the proof of (iii).

To show (iv) note that by Lemma 4.1. $A(d+1, j, 1)=A(d+1, d-j, d+1)$. If $\sigma=(d+1) \sigma(2) \ldots \sigma(d+1) \in C_{d-j, d+1}^{d}$, then the 'reverse' permutation $\widetilde{\sigma}:=$ $(d+1) \sigma(d+1) \ldots \sigma(2)$ has a descent at position 1 and whenever there is an ascent in $\sigma(2) \ldots \sigma(d+1)$. Since $\sigma$ has $d-j-1$ descents at positions $\{2, \ldots, d\}$ this implies $\operatorname{des}(\widetilde{\sigma})=1+(d-1)-(d-j-1)=j+1$, i.e. $\widetilde{\sigma} \in C_{j+1, d+1}^{d}$. We recover $\sigma$ by repeating this construction and hence $A(d+1, j, 1)=A(d+1, j+1, d+1)$.

Let $\mathcal{A}:=(A(d, i, j))_{i, j}$ be the matrix with entries $A(d, i, j)$ for fixed $d$. For pairs $(i, j),\left(i^{\prime}, j^{\prime}\right)$ we set $(i, j)<\left(i^{\prime}, j^{\prime}\right)$ if either $i<i^{\prime}$ or $i=i^{\prime}$ and $j>j^{\prime}$. This defines a total order on the set of pairs $(i, j)$. Using this ordering for the indices of the entries of the matrix we can write the matrix $\mathcal{A}$ as a vector $A(d)$.

From Corollary 4.10 and Lemma 4.1 we immediately get the following.

Corollary 4.11. The sequence $A(d)$ is unimodal and symmetric for $d \geq 1$. In particular, the peak of $A(d)$ lies in the middle.

The numerical results in Corollaries 1.3 and 4.7 suggest that the barycentric subdivision of a Cohen-Macaulay simplicial complex might be weak Lefschetz. Recall that a $(d-1)$-dimensional simplicial complex is called weak Lefschetz over $k$ if there exists an l.s.o.p. $\Theta$ for $k[\Delta]$ and a degree one element $\omega \in(k[\Delta] / \Theta)_{1}$ such that the multiplication maps

$$
\omega:(k[\Delta] / \Theta)_{i} \longrightarrow(k[\Delta] / \Theta)_{i+1}
$$

have full rank for every $i$. In particular, in our case this means injections for $0 \leq i<\frac{d}{2}$ and surjections for $\left\lceil\frac{d}{2}\right\rceil \leq i \leq \operatorname{dim} \Delta$.

Conjecture 4.12. Let $\Delta$ be a $(d-1)$-dimensional Cohen-Macaulay simplicial complex and $k$ be an infinite field. Then the barycentric subdivision of $\Delta$ is weak Lefschetz over $k$. 


\section{ACKNOWLEDGMENT}

We are grateful to Volkmar Welker, and to Isabella Novik, for helpful suggestions concerning earlier versions of this paper. Further thanks go to Mike Stillman for pointing out a mistake in an earlier version. We would also like to thank an anonymous referee whose detailed comments greatly helped to improve the presentation.

\section{REFERENCES}

1. Kenneth Baclawski and Adriano M. Garsia. Combinatorial decompositions of a class of rings. Adv. in Math., 39(2):155-184, 1981. MR609203(84a:06003a)

2. Francesco Brenti and Volkmar Welker. Conjectures on simplicial complexes and real rooted polynomials. In preparation.

3. Francesco Brenti and Volkmar Welker. $f$-vectors of barycentric subdivisions. Math. Z 259(4):849-865, 2008. MR 2403744

4. Winfried Bruns and Jürgen Herzog. Cohen-Macaulay Rings, volume 39 of Cambridge Studies in Advanced Mathematics. Cambridge University Press, Cambridge, 1993.

5. Günter Ewald and Geoffrey C. Shephard. Stellar subdivisions of boundary complexes of convex polytopes. Math. Ann., 210:7-16, 1974. MR0350623(50:3115)

6. Dominique Foata and Marcel-P. Schützenberger. Théorie Géométrique des Polynômes Eulériens. Lecture Notes in Mathematics, Vol. 138. Springer-Verlag, Berlin, 1970. MR0272642 $(42: 7523)$

7. Francis S. Macaulay. Some properties of enumeration in the theory of modular systems. Proc. London Math. Soc., 26:531-555, 1927.

8. Peter McMullen. The numbers of faces of simplicial polytopes. Israel J. Math., 9:559-570, 1971. MR0278183 (43:3914)

9. Satoshi Murai. Algebraic shifting of strongly edge decomposable spheres. math. arXiv:0709.4518, 2007.

10. Eran Nevo. Higher minors and Van Kampen's obstruction. Math. Scand. 101(2):161-176, 2007. MR2379282

11. Eran Nevo. Algebraic shifting and f-vector theory. Ph.D. thesis, The Hebrew University of Jerusalem, math arXiv:0709.3265, 2007.

12. Louis W. Shapiro, Wen Jin Woan, and Seyoum Getu. Runs, slides and moments. SIAM J. Algebraic Discrete Methods, 4(4):459-466, 1983. MR721616 (85d:05020)

13. Richard P. Stanley. The number of faces of a simplicial convex polytope. Adv. in Math., 35(3):236-238, 1980. MR 563925 (81f:52014)

14. Richard P. Stanley. Combinatorics and Commutative Algebra, volume 41 of Progress in Mathematics. Birkhäuser Boston Inc., Boston, MA, second edition, 1996. MR1453579 (98h:05001)

15. Ed Swartz. $g$-elements, finite buildings and higher Cohen-Macaulay connectivity. J. Combin. Theory Ser. A, 113(7):1305-1320, 2006. MR2259062 (2007i:05192)

16. Günter M. Ziegler. Lectures on polytopes, volume 152 of Graduate Texts in Mathematics. Springer-Verlag, New York, 1995. MR1311028(96a:52011)

Fachbereich Mathematik und Informatik, Philipps-Universität Marburg, 35032 MarBURG, Germany

E-mail address: kubitzke@mathematik.uni-marburg.de

Department of Mathematics, Cornell University, Ithaca, New York 14853

E-mail address: eranevo@math.cornell.edu 